\title{
FILTERS. THE NUMBER OF CHANNELS THAT CAN CLOG IN A NETWORK*
}

\author{
GUIDO KAMPEL ${ }^{\dagger}$ AND GUILLERMO H. GOLDSZTEIN ${ }^{\dagger}$
}

\begin{abstract}
We model filters as two-dimensional networks of channels. As a suspension (fluid with particles) flows through the filter, particles clog channels. We assume that there is no flow through clogged channels. In this paper, we compute a sharp upper bound on the number of channels that can clog before fluid can no longer flow through the filter.
\end{abstract}

Key words. filters, porous media, networks, clogging, planar graphs, Euler formula

AMS subject classification. $76 \mathrm{~S} 05$

DOI. $10.1137 / 080723703$

1. Introduction. A porous medium is a material that contains relatively small spaces filled with fluid embedded in a solid matrix. These fluid-filled spaces are called pores. A porous material is said to be permeable if fluid can flow through its pores from one end to an opposite end of the material. Filters are examples of porous materials.

Fluid suspensions (or suspensions, for short) are fluids with small solid particles in them. According to their size and properties, these particles are called fines or colloids. As a suspension flows through a permeable porous material, some fines are trapped within the material. In fact, the function of the filters we consider in this paper is to clean suspensions by capturing most particles bigger than a certain size.

The removal of particles from fluid suspensions is of importance in a wide range of industrial and technological applications such as waste water treatment [18] and other filtration processes $[4,31]$. Our studies are motivated by the filters used in the process known as deep bed filtration. As a suspension flows through a filter composed of granular or fibrous materials, fines or colloidal particles penetrate the filter and deposit at various depths [32]. As a result, the fluid suspension is cleaner when it exits the filter (i.e., it exits the filter with many fewer solid particles than it originally had when it entered the filter).

Theoretical models to study transport in porous media can be classified as either macro-scale $[5,15,22,23,24,26,32]$ or pore-scale $[9,19,28]$ models. Within the latter group, the class of network models, in which the pore space is modeled as a network of channels, is very popular. Network models provide flexibility in modeling different geometries of pore space while keeping the computational cost low. Our work belongs to this class of models.

Network models to study transport in porous media were introduced by Fatt in $1956[10,11,12]$. Donaldson, Baker, and Carrol in 1977 [8] were the first to use networks to study particle transport within porous media. The clogging of particles has been studied in networks with different geometries including bundles of parallel tubes [8], square networks $[14,16,21]$, triangular networks [3, 25], cubic networks

\footnotetext{
${ }^{*}$ Received by the editors May 8, 2008; accepted for publication (in revised form) August 26, 2008; published electronically December 17,2008 . This research was supported by the NSF.

http://www.siam.org/journals/siap/69-3/72370.html

†School of Mathematics, Georgia Institute of Technology, Atlanta, GA 30332-0160 (kampel@ math.gatech.edu, ggold@math.gatech.edu).
} 
$[2,17,29]$, bubble models $[6,20]$, and the so-called three-dimensional physically representative networks $[1,30]$.

Consider a filter that is a network of channels. As a suspension flows through the filter, particles clog channels. Assume that the suspension cannot flow through clogged channels. In this work, channels that are not clogged are called open. Note that there can be flow only through channels that are part of a percolating path of open channels, i.e., a path of channels that are not clogged connecting one side of the filter with the opposite side. As channels clog, some percolating paths of open channels are broken. Thus, suspension stops flowing not only through the clogged channels, but also through other channels, i.e., those that are no longer part of a percolating path of open channels. Thus, the filter will stop being permeable after not all, but only a number, of its channels clog. In this paper we find an upper bound of this number. Our upper bound is a function of the geometry of the network. In particular, we are able to identify the filter geometries for which the largest fraction of channels may be clogged before the filter ceases to be permeable. Our results suggest that filters with these geometries may have longer lives than others.

Our work is novel. Most of the work that can be found in the literature consists of simulations of the suspension dynamics within the medium. Our work is an analysis that is independent of the dynamics; it depends only on the topology of the network. On the other hand, our work has connections to, but also key differences from, the theory of bond percolation [13, 27]. In percolation theory, channels or edges are removed randomly and independently of each other. Here, channels clog, but neither randomly nor independently of each other; the order in which they clog is important. Nevertheless, we are able to use graph theory techniques that are also used in percolation theory.

We remark that in this paper we assume the porous media to be two-dimensional. Extensions to three-dimensional media, which could lead to results more relevant to real applications, are currently being pursued and will be presented elsewhere. We also acknowledge that our work ignores the dynamics and does not resolve the mechanisms of clogging, which would involve a variable flow-field, drag forces, and particle-solid interaction forces that are all part of a well-developed filtration theory.

This paper is organized as follows. In section 2, we describe the filters as networks. In section 3, we review the basics of graph theory that are needed in the rest of this paper. In section 4 , we obtain our upper bound. In section 5 , we show that our upper bound is sharp. In section 6 , we consider large filters and obtain an alternative description of our bound in terms of the average degree of the network. In section 7, we consider a special class of filters for which our bound is realized. In section 7, we also consider some examples and obtain some conclusions.

2. The model. We model filters as two-dimensional networks of channels as we illustrate in Figure 1. The pores are the interiors of the channels. Our filters have a bottom boundary at $y=y_{b}$ and a top boundary at $y=y_{t}$.

In our model, channels are either open or clogged. Suspension can flow only through open channels. There is no flow through clogged channels. Within an open channel, suspension flows from the end with higher pressure to the opposite end. If both ends are at the same pressure, there is no flow within the channel.

We assume that suspension can flow into the filter only through the bottom boundary and can flow out of the filter only through the top boundary. Both fluid and particles are incompressible, and thus a volume of suspension enters the filter through the bottom boundary at the same rate it exits the filter through the top boundary. 


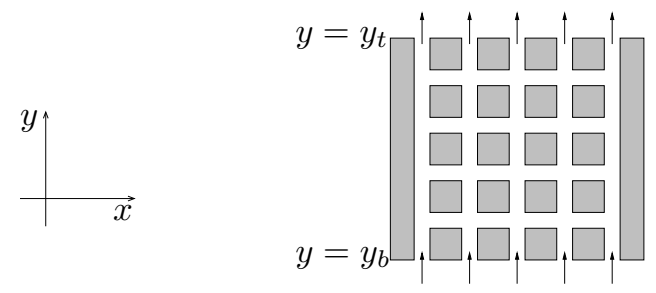

FIG. 1. As illustrated in the left figure, we use the standard notation of $x$-axis and $y$-axis for the horizontal and vertical axes, respectively. The right figure shows a network of channels. The arrows indicate the direction of the flow.

We assume that the bottom boundary is held at constant pressure $p=p_{b}$ and the top boundary at $p=p_{t}$, where $p_{b}>p_{t}$. Note that the filter is permeable if and only if there is a path of open channels connecting the bottom boundary with the top boundary. Due to the difference in pressure between the top and bottom boundaries, there is flow through the filter if and only if the filter is permeable.

We assume that initially all the channels are open. As suspension flows through the filter, particles are trapped, causing channels to clog; i.e., channels change from open to clogged. Eventually, the filter is no longer permeable. Note that an open channel can clog only if there is flow through it. For any given filter, we will find an upper bound on the number of channels that may clog under the assumption that different channels do not clog simultaneously.

Assumptions 2.1. For future reference, we list here the key assumptions of our model:

1. Channels are either open or clogged.

2. There is no flow through clogged channels.

3. Suspension can only flow into the filter through the bottom boundary and out of the filter through the top boundary.

4. Fluid and particles are incompressible.

5. Initially all the channels are open.

6. An open channel may clog if there is flow through it.

7. An open channel does not clog if there is no flow through it.

8. Different channels do not clog simultaneously.

3. Review of concepts in graph theory. In this section we review concepts of graph theory that we need in the rest of the paper. More details on graph theory can be found in [7].

A graph $G$ consists of a nonempty set of elements, called vertices or nodes, and a list of unordered pairs of these elements, called edges. It is convenient and a common practice to draw graphs in the plane. Each node is a different point in the plane, and each edge a line joining its two nodes without intersecting any other node. If $e$ is an edge joining the two nodes $a$ and $b$, we say that $a$ and $b$ are the end points of $e$ and that $e$ connects $a$ and $b$. For convenience we take $e$ (the drawing of $e$ really) to be a closed set; i.e., $e$ includes its end points. If $a=b$, i.e., the end points of an edge $e$ are the same, we say that $e$ is a loop. In a graph, two different edges do not have the same pair of end points. We have a multigraph when this restriction is removed; i.e., in a multigraph, two different edges can have the same end points.

We say that two nodes $a$ and $b$ are connected if there exists a sequence of nodes $n_{0}, n_{1}, \ldots, n_{k}$ such that $a=n_{0}, b=n_{k}$ and for each $1 \leq i \leq k$ there exists an edge $e_{i}$ 
that connects $n_{i-1}$ and $n_{i}$. In this case, the alternating sequence of nodes and edges $n_{0}, e_{1}, n_{1}, e_{2}, n_{2}, \ldots, e_{k}, n_{k}$ forms a walk between $a$ and $b$ or simply a walk. We say that $a=n_{0}$ and $b=n_{k}$ are the end points of the walk. If $n_{i} \neq n_{j}$ for all $i \neq j$, we say that the walk is a path. If $n_{0}=n_{k}$ and $n_{i} \neq n_{j}$ for $i<j$ except when $(i, j)=(0, k)$, we say that the walk is a cycle. We will identify each walk with the curve in the plane formed by its edges.

Let $G$ be a multigraph. $S$ is a submultigraph of $G$ if $S$ is a multigraph and $S$ is included in $G$, i.e., every node of $S$ is also a node of $G$ and every edge of $S$ is also an edge of $G$.

A multigraph is connected if there is a walk between any pair of its nodes, and disconnected otherwise. Every multigraph is the disjoint union of connected submultigraphs. Each of these submultigraphs is called a connected component of the multigraph.

A multigraph is planar if it can be drawn in the plane in such a way that any two different edges may intersect at only one or two of their end points. Any such drawing is a plane drawing of the multigraph. In this paper we will need to consider only planar multigraphs. We identify each planar multigraph with one of its plane drawings. In the rest of this paper, any multigraph that we mention or consider is a planar multigraph.

A multigraph divides the plane into regions called faces. More precisely, the faces are the connected components of what is left from the plane once we remove the multigraph from the plane. In other words, the faces are the connected components of the set of points in the plane that do not belong to any edge of the multigraph. Note that the faces are open sets. Any finite multigraph has an unbounded face surrounding it, called the infinity face.

Note that the boundary of any bounded face contains a cycle. Thus, a connected multigraph with no cycles has only one face, the infinity face.

Let $G$ be a multigraph. We denote by $n_{G}$ its number of nodes, by $e_{G}$ its number of edges, by $f_{G}$ its number of faces, and by $\ell_{G}$ its number of connected components. The well-known Euler formula states that

$$
n_{G}+f_{G}=e_{G}+\ell_{G}+1
$$

The degree of a node $n$, which we denote by $d_{n}$, is the number of edges that have $n$ as an end point, where the loops are counted twice. The average degree of a multigraph $G$, which we denote by $d_{G}$, is defined as the average of the degrees of the nodes of $G, d_{G}=n_{G}^{-1} \sum d_{n}$, where the sum is over all nodes $n$ and $n_{G}$ is the number of nodes of $G$. Note that

$$
d_{G}=2 \frac{e_{G}}{n_{G}},
$$

where $e_{G}$ is the number of edges of $G$. An example of a multigraph that is actually a graph is shown in Figure 2.

\section{Upper bound on the number of clogged channels.}

4.1. Microstructure of the filters. To each filter we associate a multigraph in a natural way. The edges are the channels and the nodes the end points of the edges.

Recall that the bottom and top boundaries of the filter are located at $y=y_{b}$ and $y=y_{t}$, respectively. Thus, the multigraph is included in $y_{b} \leq y \leq y_{t}$. Note that there 


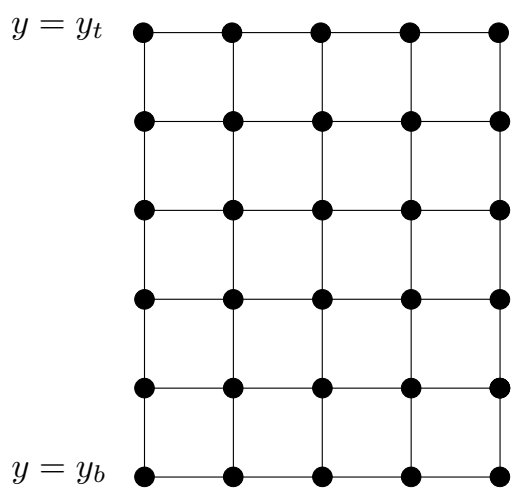

FIG. 2. Multigraph $G$. The black small circles are the nodes of the multigraph $G$ and the solid lines its edges.

are nodes in the bottom and top boundaries. For convenience, we also include edges in $y=y_{b}$ connecting the nodes in the bottom boundary. In other words, there is a path of edges in $y=y_{b}$ connecting the leftmost node in the bottom boundary with the rightmost node in that boundary. Analogously, we include edges in $y=y_{t}$ so that there is a path of edges in $y=y_{t}$ connecting the leftmost node in the top boundary with the rightmost node in that boundary.

We consider filters with a finite number of channels, and thus our multigraphs are finite multigraphs; i.e., they contain a finite number of nodes and edges. As an example, in Figure 2 we show the multigraph $G$ associated with the filter of Figure 1.

DeFINITION 4.1. We say that a node is an exterior node if it is located at $y=y_{b}$ or $y=y_{t}$. Otherwise, we say that the node is an interior node.

We also say that an edge is an exterior edge if it is included in $\left\{y=y_{b}\right\} \cup\left\{y=y_{t}\right\}$. Otherwise, we say that the edge is an interior edge.

Note that, by construction, each exterior node is the end point of at least one interior edge.

4.2. Clogged edges. The suspension enters the network through exterior nodes at $y=y_{b}$ and exits the network through exterior nodes at $y=y_{t}$.

We say that an edge is clogged if the corresponding channel is clogged. Note that the exterior edges were included for convenience; they do not correspond to any channel. Thus, we assume that there is no flow within them and that they never clog; i.e., they are always open.

As we said in section 2, we assume that initially all the edges are open and that, as suspension flows through the filter, edges clog, but different edges do not clog simultaneously.

We study our filter at a fixed time. In other words, when we say that an edge is clogged, we mean that the edge is clogged at that fixed time. Analogously, when we say that an edge is open, we mean open at that fixed time.

4.3. Mass conservation. In this subsection we introduce a definition and an observation that we will need later in the paper. This observation is a consequence of the law of mass conservation. Here, as in the rest of this section, $G$ is a fixed multigraph that corresponds to one of our filters, such as the one in Figure 2. 


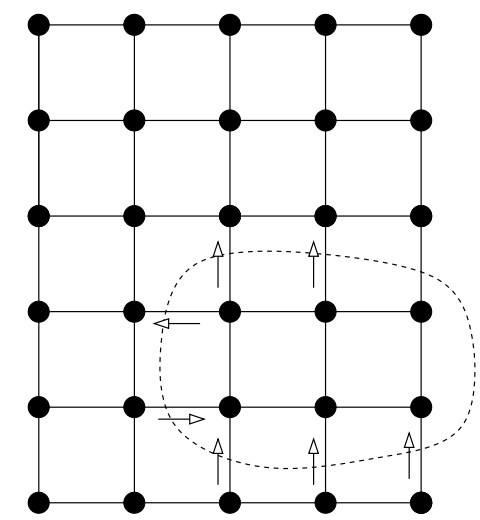

FIG. 3. $\Omega$ is the bounded open set whose boundary, $\partial \Omega$, is in dashed lines. An arrow next to an edge that intersects $\partial \Omega$ indicates the direction of the flow within that edge. No arrow next to an edge that intersects $\partial \Omega$ indicates that there is not flow through that edge. $E_{\Omega}^{\text {in }}$ is the set of edges that have arrows next to them pointing into $\Omega . E_{\Omega}^{\text {out }}$ is the set of edges that have arrows next to them pointing out of $\Omega$.

Definition 4.2. Let $\Omega$ be an open bounded set of $\mathbb{R}^{2}$ such that $\bar{\Omega}$, the closure of $\Omega$, does not intersect any exterior edge of $G$, and $\partial \Omega$, the boundary of $\Omega$, does not contain any node of $G$. We define

$$
\begin{aligned}
& E_{\Omega}=\{\text { edges in } G \text { with exactly one end point in } \Omega\}, \\
& E_{\Omega}^{\text {in }}=\left\{e \in E_{\Omega}: \text { suspension flows through e into } \Omega\right\},
\end{aligned}
$$

and

$$
E_{\Omega}^{\text {out }}=\left\{e \in E_{\Omega}: \text { suspension flows through e out of } \Omega\right\}
$$

In Figure 3 we illustrate these definitions.

Since there may be some edges without flow through them, the union of $E_{\Omega}^{\text {in }}$ and $E_{\Omega}^{\text {out }}$ need not be $E_{\Omega}$. In particular, clogged edges in $E_{\Omega}$ are neither in $E_{\Omega}^{\text {in }}$ nor in $E_{\Omega}^{\text {out }}$. Note that there may also be open edges $E_{\Omega}$ without flow through them. Thus, there may be open edges in $E_{\Omega}$ that are in neither $E_{\Omega}^{\text {in }}$ nor $E_{\Omega}^{\text {out }}$. Note also that $E_{\Omega}^{\text {in }}$ and $E_{\Omega}^{\text {out }}$ are disjoint sets.

ObSERVATION 4.1. Let $\Omega$ be an open bounded set of $\mathbb{R}^{2}$ such that $\bar{\Omega}$ does not intersect any of the exterior edges of $G$ and $\partial \Omega$ does not contain any node of $G$. Then we have the following:

1. The rate at which suspension flows into $\Omega$ through the edges in $E_{\Omega}^{\text {in }}$ is equal to the rate at which suspension flows out of $\Omega$ through the edges in $E_{\Omega}^{\text {out }}$.

2. Let $e \in E_{\Omega}$. If all the other edges in $E_{\Omega}$ are clogged, then there is no flow through e.

3. If $E_{\Omega}$ is not empty, then at least one of the edges in $E_{\Omega}$ is not clogged.

Proof. Suspension can flow into the filter only through its bottom boundary (i.e., the exterior nodes at $y=y_{b}$ ) and out of the filter only through its top boundary. Thus, since $\Omega$ does not contain any of the exterior nodes, there are neither mass sources nor mass sinks within $\Omega$. This, together with the fact that the suspension is incompressible, implies point 1. 


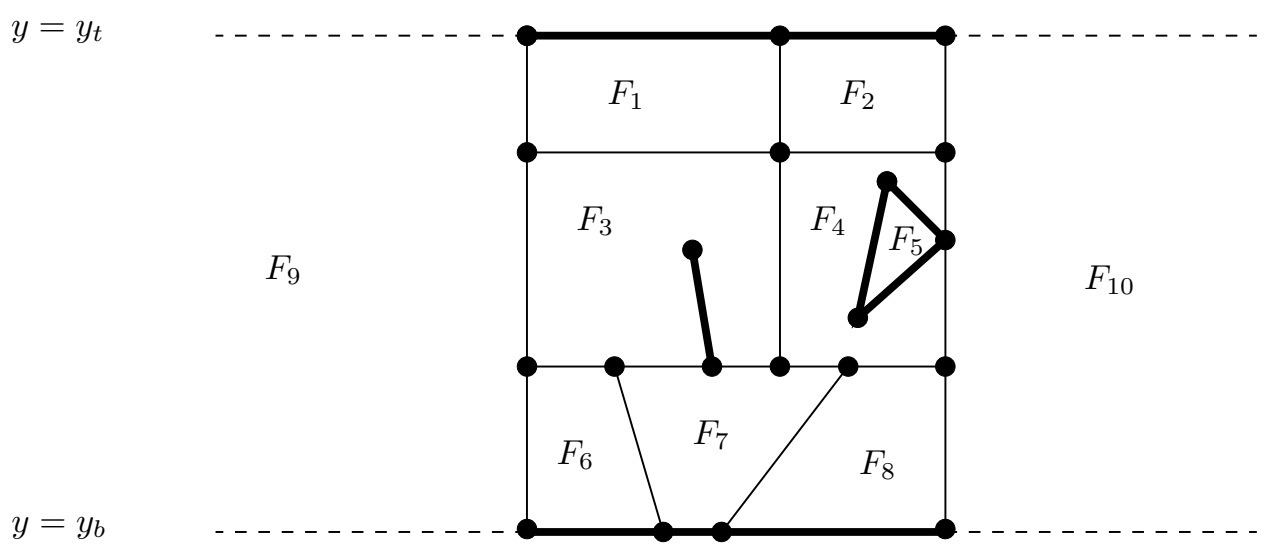

FIG. 4. Example of a multigraph $G$. The edges of $G$ are the thin and thick solid lines. The dashed lines are not part of $G . F_{i}(1 \leq i \leq 10)$ are the connected components of $\left\{y_{b}<y<y_{t}\right\}-G$. The edges in thick solid lines are not contained in any percolating path. The edges in thin solid lines are contained in percolating paths. There can be flow only through the thin edges.

Let $e$ be an edge in $E_{\Omega}$. If there is flow through $e$ into $\Omega$, point 1 implies that there should be flow out of $\Omega$ through some edge in $E_{\Omega}$ other than $e$. This is a contradiction since all the edges in $E_{\Omega}$ other than $e$ are clogged. Thus, there is no flow through $e$ into $\Omega$. A similar argument shows that there is no flow through $e$ out of $\Omega$ either, which proves point 2 .

We prove point 3 by contradiction. Assume that all the edges in $E_{\Omega}$ are clogged. Let $e$ be the edge in $E_{\Omega}$ that clogged last. Once the other edges were clogged, there was no more flow through $e$, and thus $e$ could not have clogged, because our model assumes that an open edge does not clog if there is no flow through the edge.

4.4. $C^{\star}$, a multigraph associated with the clogged edges. In this subsection we construct a multigraph $C^{\star}$ that is associated with the set of clogged edges.

We first note that the bounded connected components of the set $\left\{y_{b}<y<y_{t}\right\}-G$ are the bounded faces of $G$. In addition, $\left\{y_{b}<y<y_{t}\right\}-G$ has two unbounded connected components, one to the left of $G$ and the other to its right. An example is shown in Figure 4, where the edges of $G$ are the thin and thick solid lines. The dashed lines are not part of $G . F_{i}(1 \leq i \leq 10)$ are the connected components of $\left\{y_{b}<y<y_{t}\right\}-G$. While $F_{i}$ for $1 \leq i \leq 8$ are the bounded faces of $G, F_{9}$ and $F_{10}$ are not faces of $G$.

Before proceeding with the construction of $C^{\star}$ we first need some preliminary definitions and observations.

Definition 4.3. We say that a path $P=n_{0}, e_{1}, n_{1}, \ldots, e_{r}, n_{r}$ in $G$ is a percolating path if $n_{0}$ is a bottom exterior node, $n_{r}$ is a top exterior node, and $n_{1}, \ldots, n_{r-1}$ are interior nodes.

ObSERVATION 4.2. Let e be an edge in $G$. If there is no percolating path that contains e, then e never clogs.

The claim of Observation 4.2 is illustrated in Figure 4. Let $e$ be an edge of $G$. If there is no percolating path that contains $e$, then the pressures at the end points of $e$ are equal, which implies that there is no flow through $e$, and thus $e$ can never clog.

Note that all percolating paths split the strip $\left\{y_{b} \leq y \leq y_{t}\right\}$ into two connected components, one to the right of the path and the other to its left. 


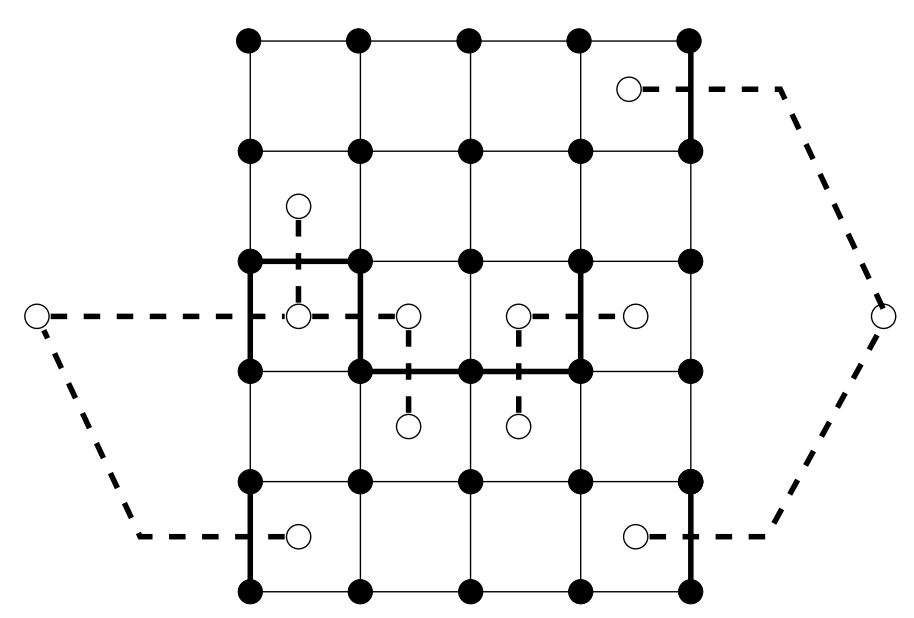

FIG. 5. The clogged edges are thick solid lines. The open edges are in thin solid lines. The thick dashed lines are the edges in $C^{\star}$. The white circles are the nodes of $C^{\star}$.

Definition 4.4. Let $P$ be a percolating path. We say that a set $S$ is to the right of $P$ if $S$ is included in the closure of the right connected component of $\left\{y_{b} \leq y \leq y_{t}\right\}-P$. Analogously, $S$ is to the left of $P$ if $S$ is included in the closure of the left connected component of $\left\{y_{b} \leq y \leq y_{t}\right\}-P$.

For example, the set $F_{10}$ in Figure 4 is to the right of any percolating path of the graph of that figure.

OBSERVATION 4.3. Let $P$ be a percolating path and $F$ a connected component of $\left\{y_{b}<y<y_{t}\right\}-G$. Then $F$ is either to the right of $P$ or to the left of $P$.

While obvious, the last observation leads to the next one that will be key in our construction of $C^{\star}$.

ObSERVATION 4.4. Let $e$ be an edge in $G$. If there is a percolating path that contains $e$, then $e$ is in the boundary of two connected components of $\left\{y_{b}<y<y_{t}\right\}-G$.

We are now ready to start our construction of a drawing of $C^{\star}$.

Select a point inside each connected component of the set $\left\{y_{b}<y<y_{t}\right\}-G$. We call this set of points $\mathcal{N}^{\star}$.

For each edge of $G$ that is clogged, we draw exactly one edge of $C^{\star}$ as follows. Let $e$ be a clogged edge of $G$. Observations 4.2 and 4.4 imply that $e$ is included in the boundary of two connected components of $\left\{y_{b}<y<y_{t}\right\}-G$. Let $a^{\star}$ and $b^{\star}$ be the points of $\mathcal{N}^{\star}$ that are included in these components. We draw exactly one edge $e^{\star}$ of $C^{\star}$ connecting $a^{\star}$ and $b^{\star}$ in such a way that $e^{\star}$ intersects $e$ in exactly one point, $e^{\star}$ does not intersect any other edge of $G$, and $e^{\star} \in\left\{y_{b}<y<y_{t}\right\}$. We say that $e^{\star}$ is the edge of $C^{\star}$ associated with $e$. This construction is carried out in such a way that edges of $C^{\star}$ may intersect only at their end points. The nodes of $C^{\star}$ are the end points of the edges in $C^{\star}$. Note that the set of nodes of $C^{\star}$ is a subset of $\mathcal{N}^{\star}$.

In Figure 5 we show an example of a set of clogged edges and the associated $C^{\star}$. The clogged edges are the thick solid lines, and the edges of $C^{\star}$ are the dashed lines. The white circles are the nodes of $C^{\star}$.

4.5. Bounding the number of clogged edges. The next sequence of observations will allow us to bound the number of clogged edges.

OBSERVATION 4.5. The number of clogged edges is equal to the number of edges in $C^{\star}$. 
This last observation is an immediate consequence of the definition of $C^{\star}$.

Observation 4.6. $C^{\star}$ does not have any bounded faces.

Proof. The proof proceeds by contradiction. Assume that $\Omega$ is a bounded face of $C^{\star}$. From the definition of $C^{\star}$, the edges of $G$ that intersect $C^{\star}$ are clogged. Thus, all the edges of $G$ that intersect $\partial \Omega$ are clogged. Note also that $\bar{\Omega}$, the closure of $\Omega$, does not intersect any of the exterior edges of $G$; $\partial \Omega$, the boundary of $\Omega$, does not contain any node of $G$; and the number of edges of $G$ that intersect $\partial \Omega$ is positive.

The above paragraph is in contradiction of point 3 of Observation 4.1. Thus, $C^{\star}$ does not have any bounded faces.

As a consequence, the only face of $C^{\star}$ is its unbounded face. Thus, we have the following.

OBSERVATION 4.7. $C^{\star}$ has only one face.

Due to the definition of $C^{\star}$, we also have the following observation.

Observation 4.8. Let $n_{C^{\star}}$ be the number of nodes of $C^{\star}$. Then, $n_{C^{\star}} \leq f_{G}+1$, where $f_{G}$ is the number of faces of $G$.

We are now ready to bound the number of clogged edges. Let $n_{C^{\star}}, e_{C^{\star}}, f_{C^{\star}}$, and $\ell_{C^{\star}}$ be the number of nodes, edges, faces, and connected components of $C^{\star}$. Euler's formula implies

$$
e_{C^{\star}}=n_{C^{\star}}+f_{C^{\star}}-\ell_{C^{\star}}-1 .
$$

From Observation 4.7 we have $f_{C^{\star}}=1$. Thus, (4.4) reduces to

$$
e_{C^{\star}}=n_{C^{\star}}-\ell_{C^{\star}} .
$$

As a consequence, using Observation 4.8, we have

$$
e_{C^{\star}} \leq f_{G}+1-\ell_{C^{\star}},
$$

where $f_{G}$ is the number of faces of $G$. Finally, since $\ell_{C^{\star}} \geq 1$ and $e_{C^{\star}}$ is the number of clogged edges, we obtain our bound, which we summarize in the following theorem.

THEOREM 4.5. Let $G$ be a multigraph that corresponds to one of our filters. Then,

$$
\#\{\text { clogged edges }\} \leq \#\{\text { faces of } G\} .
$$

5. Optimality of the bound. As always, $G$ is the multigraph of one of our filters.

Definition 5.1. We say that $e_{1}, e_{2}, \ldots, e_{s}$ is a feasible clogging sequence or (for short) feasible sequence if, for each $1 \leq i \leq s$, there is flow through the edge $e_{i}$ when $e_{1}, e_{2}, \ldots, e_{i-1}$ are clogged and all the other edges are open. We say that $s$ is the length of the sequence.

Recall that an edge can clog only when suspension flows through it. Thus, if $q$ edges clogged, and the $i$ th edge that clogged was $e_{i}$, then $e_{1}, e_{2}, \ldots, e_{q}$ is a feasible sequence of edges. Note that the bound of section 4 is actually a bound on the length of feasible sequences of edges.

While there are many feasible sequences that make the filter nonpermeable, only one actually realizes. The flow conditions, conductivity of the channels, as well as other factors determine the feasible sequence that realizes, which generally has fewer edges than other feasible clogging sequences. It is not our goal to find the sequence that realizes. In this section we show that, if every interior edge of $G$ is contained in a 

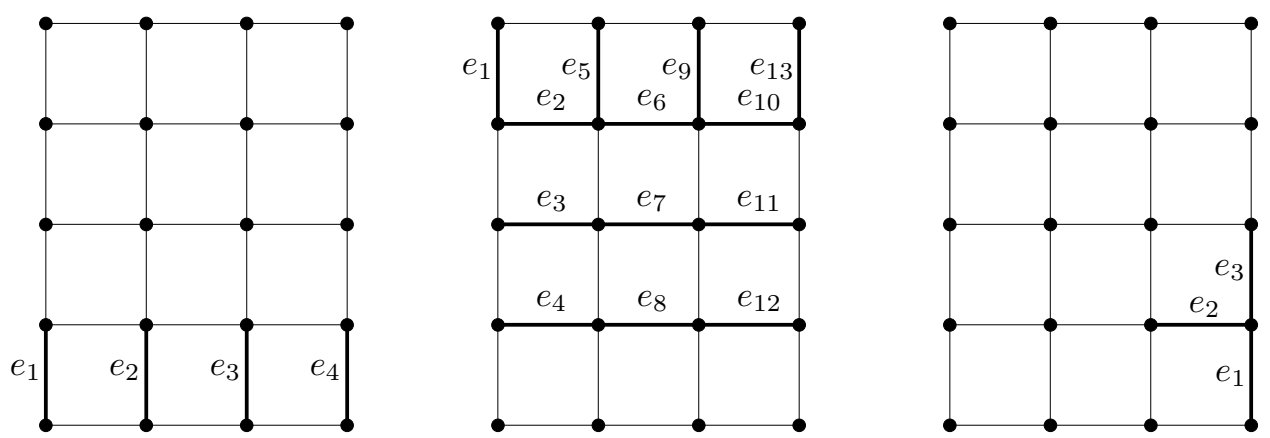

FIG. 6. The sequences $e_{1}, \ldots, e_{r}$ in the left and middle figures $(r=4$ in the left figure and $r=13$ in the middle figure) are two feasible sequences of edges that make the filter nonpermeable. Note that the number of faces of the graph is 13 . Thus, the sequence of the middle figure is of maximum length. Our work does not predict whether the sequence in the left figure, the one in the middle, or another sequence is realized; thus, we can predict only that the sequence that realizes has length less than or equal to 13. The sequence in the right figure is not feasible. Once $e_{1}$ and $e_{2}$ clog, there is no more flow through $e_{3}$, and thus it cannot clog.

percolating path, our bound is sharp in the sense that there exists a feasible sequence of edges whose length is equal to our bound, the number of faces of $G$ (the right-hand side of (4.7)). However, it should be noted that the length of the feasible sequence that actually realizes and makes the filter nonpermeable may be smaller. In other words, while the length of the longest feasible sequence of edges is an upper bound on the number of channels that actually clog, these numbers may not be equal. Illustrative examples are given in Figure 6. We will come back to this issue in section 7.

Let $e$ be an interior edge of $G$. As illustrated in Figure 4 and previously discussed, if there is no percolating path that contains $e$, then the pressure at the end points of $e$ are equal, which implies that there is no flow through $e$ and thus that $e$ can never clog. Removing first all such edges from $G$, then all the exterior edges, then the nodes that are left isolated, and finally adding new exterior edges as necessary leads to a new multigraph $\tilde{G}$ for which the bound will be attained. Note that the flow in $G$ is exactly equal to the flow in $\tilde{G}$. There is no flow within edges of $G$ that do not belong to $\tilde{G}$.

\subsection{Leftmost percolating paths.}

ObSERVATION 5.1. Let $P=n_{0}, e_{1}, n_{1}, \ldots, e_{r}, n_{r}$ be a percolating path. Let e be an edge to the left of $P$. If $e$ is included in a percolating path, then there exists a percolating path $R$ such that $P$ and $e$ are to the right of $R$.

Proof. Assume that $e$ is not in $P$, since otherwise the observation is trivially true by selecting $R=P$. Let $Q$ be a percolating path that contains $e$. Let $\bar{Q}$ be the largest path that satisfies (1) $\bar{Q}$ is included in $Q,(2) \bar{Q}$ contains $e,(3) \bar{Q}$ is to the left of $P$, and (4) $\bar{Q}$ may intersect $P$ only at the end points of $\bar{Q}$.

If $\bar{Q}=Q$, as in Figure $7($ a), select $R=Q$. Note that $e$ and $P$ are to the right of $R$. Otherwise, $\bar{Q}$ intersects $P$. In this case, $\bar{Q} \cap P$ splits $P$ into two or three connected sections. Replacing one of these connected sections with $\bar{Q}$ leads to the percolating path $R$ we are looking for. If $\bar{Q}$ contains a bottom exterior node, as in Figure 7(b), we replace the section of $P$ that has a bottom exterior node. If $\bar{Q}$ contains a top exterior node, as in Figure 7(c), we replace the section of $P$ that has a top exterior node. If $\bar{Q}$ does not contain any exterior node, as in Figure $7(\mathrm{~d})$, we replace the section of $P$ 


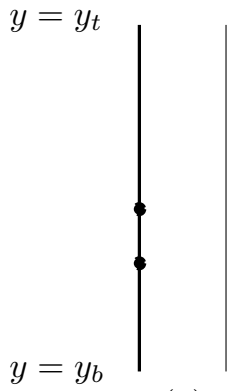

(a)

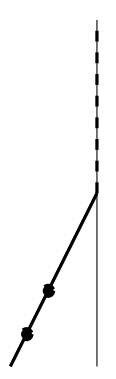

(b)

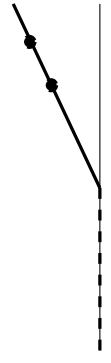

(c)

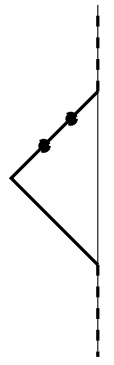

(d)

FIG. 7. The four different possibilities of Observation 5.1. We do not show all the multigraph $G$, only e, $\bar{Q}$, and $P$. The edge $e$ is the segment between the solid small circles. $P$ is the thin solid vertical line, $\bar{Q}$ the thick solid line, and $R$ the union of $\bar{Q}$ and the dashed lines.

without exterior nodes.

This last observation and the fact that each exterior node is the end point of an interior edge lead to the following.

OBSERVATION 5.2. If every interior edge in $G$ is included in a percolating path, then there is a unique percolating path $P$ in $G$ such that $G$ is to the right of $P$. We call $P$ the leftmost percolating path of $G$.

5.2. A feasible sequence of maximum length. The first edge. Assume that every interior edge in $G$ is included in a percolating path. Our goal is to construct a feasible sequence of edges $e_{1}, e_{2}, \ldots, e_{N}$ of maximum length. Let $P$ be the leftmost percolating path of $G$. In this subsection we identify $\bar{P}$, a subpath of $P$, from which $e_{1}$ will be selected. The selection of $\bar{P}$ is done with care so that the rest of the sequence, $e_{2}, \ldots, e_{N}$, can be constructed inductively, as we will do in the next subsection.

OBSERVATION 5.3. Assume that every interior edge in $G$ is included in a percolating path of $G$. Let $P$ be the leftmost percolating path of $G$. Assume that there are no bounded faces $F$ of $G$ such that $\partial F \cap P$ contains an edge. Then $P=G$.

Proof. The proof is by contradiction. Assume $P \neq G$. Then there exists an edge in $G$ not in $P$. In fact, since every exterior node is the end point of an interior edge, we have that there exists an interior edge $e$ in $G$ such that $e$ is not in $P$. Let $Q$ be a percolating path in $G$ containing $e$. Note that there is a nonempty open bounded $\Omega$ enclosed by $P, Q, y=y_{b}$, and $y=y_{t}$. Note also that at least one edge of $P$ is in the boundary of $\Omega$. The closure of $\Omega$ is the union of the closure of the bounded faces of $G$ included in $\Omega$. Thus, there exists $F$, a bounded face of $G$, such that $\partial F \cap P$ contains an edge. This is a contradiction, which proves the observation.

ObSeRvation 5.4. Assume that every interior edge in $G$ is included in a percolating path of $G$. Let $F$ be a bounded face of $G$. Then, $\partial F \cap\left\{y=y_{b}\right\}$ is connected, and $\partial F \cap\left\{y=y_{t}\right\}$ is also connected.

Proof. Assume that $\partial F \cap\left\{y=y_{b}\right\}$ is not connected. Then, as illustrated in Figure 8, there is a path $\bar{Q}$ that is included in $\partial F$ such that $\bar{Q} \cap\left\{y=y_{b}\right\}$ are the end points of $\bar{Q}$, the edges in $\bar{Q}$ are interior edges, and none of them is included in a percolating path of $G$, which contradicts our assumption. Thus, $\partial F \cap\left\{y=y_{b}\right\}$ is connected. Analogously, $\partial F \cap\left\{y=y_{t}\right\}$ is also connected.

ObSERVATION 5.5. Assume that every interior edge in $G$ is included in a percolating path of $G$. Let $P$ be the leftmost percolating path of $G$. Assume that $G$ has a 


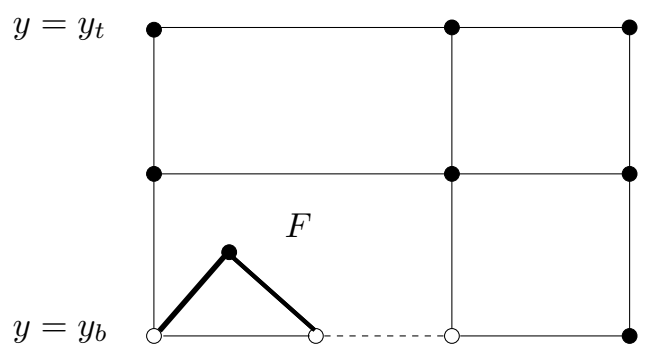

FIG. 8. Multigraph G. $\partial F \cap\left\{y=y_{b}\right\}$ is the edge in dashed line and the white nodes, which is a disconnected set. The thick solid line is $\bar{Q}$. The edges in $\bar{Q}$ are not included in any percolating path of $G$.

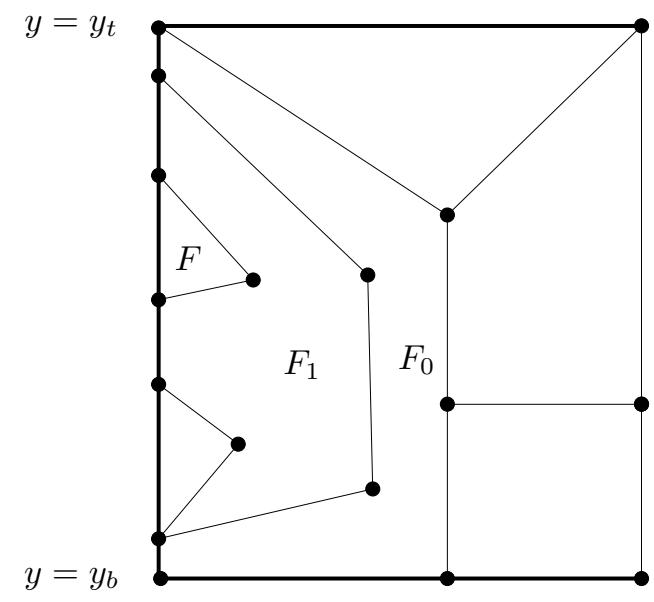

FIG. 9. Multigraph $G$. The edges of the path $S$ are in thick lines. Example of a sequence $F_{0}, F_{1}, F_{2}=F$ constructed as in Observation 5.5.

bounded face. Then, there exists $F$, a bounded face of $G$, such that

1. $\partial F \cap P$ contains an edge, and

2. $\partial F \cap\left(P \cup\left\{y=y_{t}\right\} \cup\left\{y=y_{b}\right\}\right)$ is connected.

Proof. Let $S$ be the path that results from the following steps. We start at the rightmost exterior node of the bottom boundary and walk left along that boundary toward the path $P$. We continue walking through $P$ to the top boundary. We then walk right along the top boundary and end the path at the rightmost exterior node of the top boundary (see Figure 9).

Since $G$ has a bounded face, $P \neq G$, and thus, from Observation 5.3, there exists $F_{0}$, a bounded face of $G$, such that $\partial F_{0} \cap P$ contains an edge. Let $S_{F_{0}}=\partial F_{0} \cap S$ and $\tilde{S}_{F_{0}}$ be the smallest path included in $S$ that contains $S_{F_{0}}$. Recall that paths are connected. Thus, if $\tilde{S}_{F_{0}}=S_{F_{0}}$, then $F=F_{0}$ is a face we are looking for.

We next show that $P \cap \tilde{S}_{F_{0}} \subseteq S_{F_{0}}$ implies that $\tilde{S}_{F_{0}}=S_{F_{0}}$. Assume that $P \cap \tilde{S}_{F_{0}} \subseteq S_{F_{0}}$. If both $\partial F_{0} \cap\left\{y=y_{b}\right\}$ and $\partial F_{0} \cap\left\{y=y_{t}\right\}$ are empty sets, then $\tilde{S}_{F_{0}}=P \cap \tilde{S}_{F_{0}} \subseteq S_{F_{0}}$. Assume now that $\partial F_{0} \cap\left\{y=y_{b}\right\}$ is nonempty. Thus, $\tilde{S}_{F_{0}}$ contains the exterior node of $P$ in the bottom boundary, and since $P \cap \tilde{S}_{F_{0}} \subseteq S_{F_{0}}$, that exterior node is also in $S_{F_{0}}$. Thus, $\left\{y=y_{b}\right\} \cap S_{F_{0}}=\partial F_{0} \cap\left\{y=y_{b}\right\}$ also contains that exterior node and, given that $\partial F_{0} \cap\left\{y=y_{b}\right\}$ is connected due to Observation 5.4, 


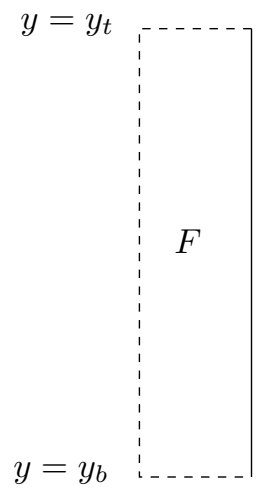

(a)

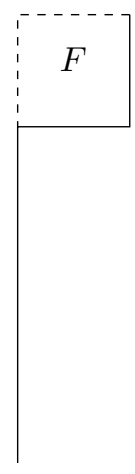

(b)

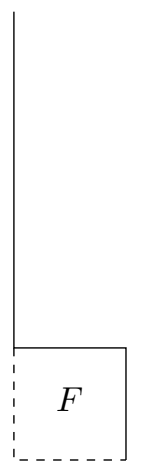

(c)

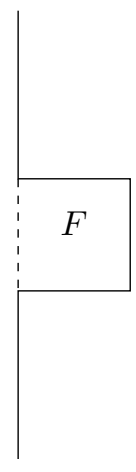

(d)

Fig. 10. Four different possibilities of $F$ from Observation 5.6 and Theorem 5.2. The solid line is the percolating path $S$ of Observation 5.6. The left vertical line is $P . P \cap S$ is in solid line, and the section of $P$ that does not intersect $S$ is in dashed line. The dashed horizontal lines are the sections of the top and bottom boundary that are to the left of $S$.

we have that $\left\{y=y_{b}\right\} \cap \tilde{S}_{F_{0}} \subseteq S_{F_{0}}$. This argument applied to the top boundary leads to $\tilde{S}_{F_{0}}=\left(P \cup\left\{y=y_{t}\right\} \cup\left\{y=y_{b}\right\}\right) \cap \tilde{S}_{F_{0}} \subseteq S_{F_{0}}$ if $\left(P \cap \tilde{S}_{F_{0}}\right) \subseteq S_{F_{0}}$.

We are left to show that the observation is true when $\tilde{S}_{F_{0}} \neq S_{F_{0}}$ (see Figure 9), and so we now assume $\tilde{S}_{F_{0}} \neq S_{F_{0}}$. Given the above paragraph, we have that $\tilde{S}_{F_{0}}-S_{F_{0}}$ intersects $P$ in at least one edge, say $e$. As illustrated in Figure 10, we can select $F_{1}$, a bounded face of $G$, such that $\partial F_{1}$ contains $e$. Let $S_{F_{1}}=\partial F_{1} \cap S$ and $\tilde{S}_{F_{1}}$ be the smallest path included in $S$ that contains $S_{F_{1}}$. If $\tilde{S}_{F_{1}}=S_{F_{1}}$, then $F=F_{1}$ is a face we are looking for. Otherwise, we note that $\tilde{S}_{F_{1}}$ is included in a connected component of $\tilde{S}_{F_{0}}-S_{F_{0}}$, and thus $\tilde{S}_{F_{1}} \subsetneq \tilde{S}_{F_{0}}$. As a consequence, since $G$ is a finite multigraph, repeating this procedure as many times as necessary, we will find the face $F$ that we are looking for (see Figure 9).

Assume that every interior edge in $G$ is included in a percolating path. Let $F$ be a bounded face of $G$ that satisfies the conditions of Observation 5.5. Let $\tilde{P}=P \cap \partial F$, where $P$ is the leftmost percolating path of $G$. In the next subsection we will show how to construct a feasible sequence of edges $e_{1}, e_{2}, \ldots, e_{N}$ of maximum length where $e_{1}$ will be selected from $\tilde{P}$.

\subsection{Feasible sequence of edges of maximal length.}

ObSeRvation 5.6. Assume that every interior edge in $G$ is included in a percolating path. Let $P$ be the leftmost percolating path of $G$. Assume that $G$ has a bounded face. Let $F$ be a face of $G$ such that (1) $\partial F \cap P$ contains an edge, and (2) $\partial F \cap\left(P \cup\left\{y=y_{t}\right\} \cup\left\{y=y_{b}\right\}\right)$ is connected.

Then, for every interior edge $e$ in $G$ not in $\partial F \cap P$ there exists a percolating path $Q$ of $G$ such that $Q$ contains $e$ and $Q$ does not have any edge in $\partial F \cap$ $\left(P \cup\left\{y=y_{t}\right\} \cup\left\{y=y_{b}\right\}\right)$.

Proof. Let $S_{1}$ be the section of $P$ that does not intersect $\partial F$, i.e., $S_{1}=P-\partial F$. Let $S_{2}$ be the section of $\partial F$ that intersects neither $P$ nor the boundaries, i.e., $S_{2}=$ $\partial F-\left(P \cup\left\{y=y_{t}\right\} \cup\left\{y=y_{b}\right\}\right)$. Let $S$ be the percolating path that results from walking along $S_{1} \cup S_{2}$ (see Figure 10). Note that every edge in $G$ not in $\partial F \cap\left(P \cup\left\{y=y_{t}\right\} \cup\left\{y=y_{b}\right\}\right)$ is to the right of $S$. 


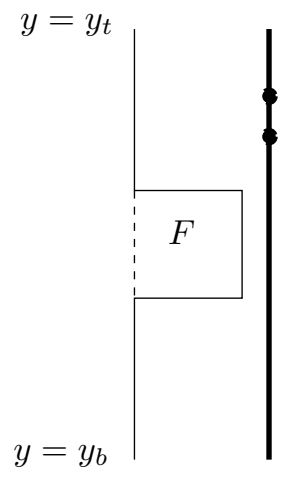

(a)

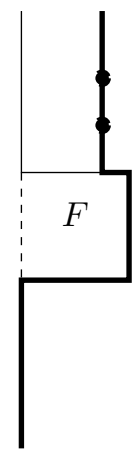

(b)

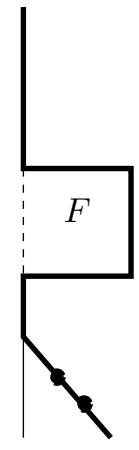

(c)

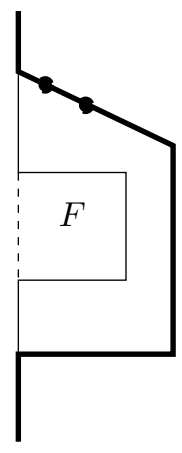

(d)

FIG. 11. Four different possibilities of the percolating path $Q$, in thick solid lines, from Observation 5.6. The edge $e$ is between the solid small circles. The left vertical line is $P$.

Let $e$ be an interior edge in $G$ not in $\partial F \cap P$. Let $R$ be a percolating path of $G$ that contains $e$. Let $\bar{Q}$ be the largest subpath of $R$ that contains $e$ such that $\bar{Q}$ may only intersect $S$ at the end points of $\bar{Q}$. As illustrated in Figure 11, we can construct a percolating path $Q$ that contains $\bar{Q}$, may contain sections of $S$, but does not contain any edge outside $\bar{Q} \cup S$. Thus, $Q$ contains $e$ and does not have any edge in $\partial F \cap\left(P \cup\left\{y=y_{t}\right\} \cup\left\{y=y_{b}\right\}\right)$.

ObSERVATION 5.7. Assume that every interior edge in $G$ is included in a percolating path of $G$. Let $P$ be the leftmost percolating path of $G$. Assume that $G$ has a bounded face. Let $F$ be a bounded face of $G$ such that (1) $\partial F \cap P$ contains an edge, and (2) $\partial F \cap\left(P \cup\left\{y=y_{t}\right\} \cup\left\{y=y_{b}\right\}\right)$ is connected. Let $G^{\prime}$ be the multigraph that results from first removing from $G$ the edges in $\partial F \cap\left(P \cup\left\{y=y_{t}\right\} \cup\left\{y=y_{b}\right\}\right)$ and then removing the nodes that are left isolated. Then the following hold:

1. Every interior edge in $G^{\prime}$ is included in a percolating path in $G^{\prime}$.

2. $f_{G^{\prime}}=f_{G}-1$; i.e., the number of faces of $G^{\prime}$ is equal to the number of faces of $G$ minus one.

3. $G^{\prime}$ is the multigraph of one of our filters.

Proof. Point 1 is an immediate consequence of Observation 5.6.

Point 2 results from the simple facts that (1) all the bounded faces of $G^{\prime}$ are bounded faces of $G$, (2) the only bounded face of $G$ that is not a face of $G^{\prime}$ is $F$, and (3) both $G$ and $G^{\prime}$ (as well as any multigraph) have only one unbounded face. (Note that $G^{\prime}$ results from removing the dashed lines in Figure 10.)

Point 3 is also clear.

THEOREM 5.2. If every interior edge in $G$ is included in a percolating path of $G$, then there exists a feasible sequence of edges of length $f_{G}$. Thus, our bound is optimal for this class of filters.

Proof. We will prove the theorem by induction on $f_{G}$. First note that $f_{G}=1$ if and only if $G$ is a percolating path. In this case, any edge of $G$ forms a feasible sequence of edges of length $f_{G}=1$.

Assume now that $f_{G}>1$. Let $P$ be the leftmost percolating path of $G$. Note that $P$ cannot be equal to $G$, since otherwise $f_{G}$ would be equal to one.

Let $F$ be a bounded face of $G$ such that (1) $\partial F \cap P$ contains an edge, and (2) $\partial F \cap\left(P \cup\left\{y=y_{t}\right\} \cup\left\{y=y_{b}\right\}\right)$ is connected. Such a face exists by the observations of this section. Let $e_{1}$ be any edge in $\partial F \cap P$. 
Let $G^{\prime}$ be the multigraph that results from first removing from $G$ the edges in $\partial F \cap\left(P \cup\left\{y=y_{t}\right\} \cup\left\{y=y_{b}\right\}\right)$ and then removing the nodes that are left isolated. From Observation 5.7, every interior edge in $G^{\prime}$ is included in a percolating path in $G^{\prime}, f_{G^{\prime}}=f_{G}-1$, and $G^{\prime}$ is the multigraph of one of our filters.

By inductive hypothesis there exists in $G^{\prime}$ a feasible sequence of edges of length $f_{G^{\prime}}=f_{G}-1$. For convenience, call one such sequence $e_{2}, \ldots, e_{f_{G}}$. From the observations of this section, it follows that $e_{1}, e_{2}, \ldots, e_{f_{G}}$ is a feasible sequence of edges in $G$, which proves the theorem.

6. The bound in terms of the average degree of $G$ for large filters. As always, $G$ is a multigraph of one of our filters. We assume in this section that every interior edge of $G$ is contained in a percolating path. We recall that $f_{G}, e_{G}$, and $n_{G}$ are the numbers of faces, edges, and nodes of $G$, respectively. We also recall that $d_{G}$, the average degree of $G$, is given by $d_{G}=2 e_{G} / n_{G}$ (see (3.2)).

Assume that the number of edges is large, i.e., $e_{G} \gg 1$. In this case, the Euler formula $f_{G}+n_{G}=e_{G}+\ell_{G}+1$ reduces to $f_{G}+n_{G} \approx e_{G}$ since $\ell_{G}=1$. Thus, from (3.2), we have $f_{G}+2 e_{G} / d_{G} \approx e_{G}$. This leads to the following observation.

OBSERVATION 6.1. If $e_{G} \gg 1$ and $d_{G} \neq 2$, then our bound (4.7) reads

$$
\#\{\text { clogged edges }\} \lesssim \frac{d_{G}-2}{d_{G}} e_{G} .
$$

In particular, if every interior edge of $G$ is contained in a percolating path, the number of edges in a feasible sequence of edges with maximum length is asymptotically $\left(\left(d_{G}-2\right) / d_{G}\right) e_{G}$.

In many situations of interest, $G$ is a graph; i.e., no two edges have the same end points. For example, if all the edges in a multigraph are straight segments, then the multigraph is really a graph. It is a well-known fact from graph theory that, if $G$ is a planar graph, the average degree of $G$ is bounded by 6 , i.e., $d_{G} \leq 6$. This leads to the following observation.

OBSERVATION 6.2. If $G$ is a graph and $e_{G} \gg 1$, then

$$
\#\{\text { clogged edges }\} \lesssim \frac{2}{3} \#\{\text { all edges }\}
$$

A natural goal is to design filters that use as much of the pore space as possible to trap particles before the filter ceases to be permeable. Thus, of particular interest is to know the proportion of channels that are clogged when the filter ceases to be permeable. The last observation provides a bound on this quantity whenever $G$ is a graph.

As particular examples, consider the graphs of Figure 12. At this point do not make a distinction between solid and dashed edges. In the large filter limit, i.e., the distance between the top and bottom boundaries is much larger than the length of the edges, the graph with square bounded faces satisfies $d_{G} \approx 4$, and thus, for this graph, (6.1) implies \#\{clogged edges $\} \lesssim e_{G} / 2$. For the graph with triangle bounded faces, we have $d_{G} \approx 6$ and thus $\#\{$ clogged edges $\} \lesssim 2 e_{G} / 3$. For the graph with hexagonal bounded faces, we have $d_{G} \approx 3$ and thus $\#\{$ clogged edges $\} \lesssim e_{G} / 3$.

7. A subclass of filters and examples. In this section, we consider filters in which every interior edge is included in a percolating path. We have shown that, for this kind of filters, our bound is sharp; i.e., there is a feasible sequence of edges whose number of edges or length is equal to our bound, i.e., the number of faces of 

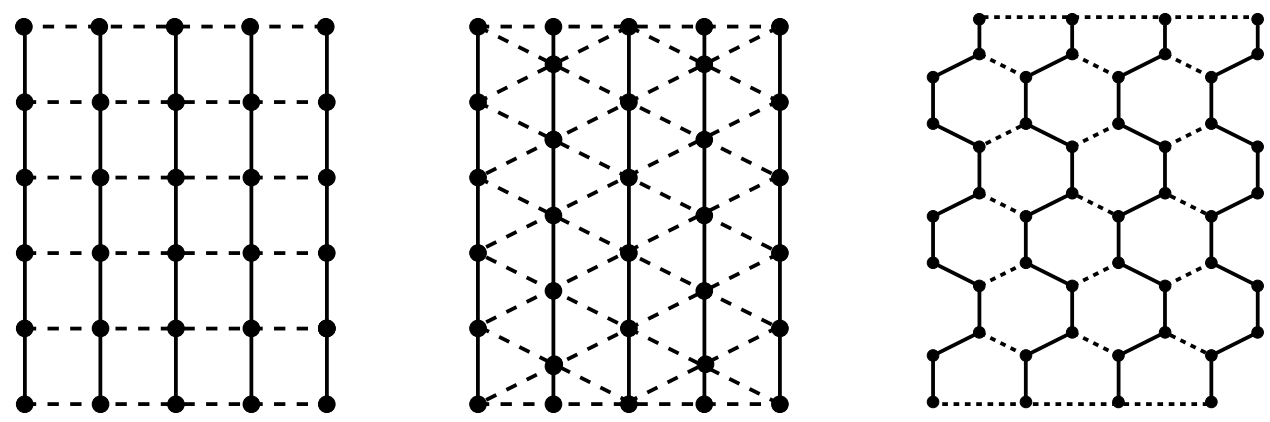

FIG. 12. Multigraphs with geometries of class $\mathcal{A}$. The percolating paths $P_{i}$ are in solid lines. The crossing paths $H_{i, j}$ are in dashed lines.

the multigraph (see the right-hand side of (4.7)). However, in general, there are many feasible sequences of edges that make the filter nonpermeable, and the length of most of them is less than our bound. Thus, the feasible sequence of edges that is realized, i.e., the sequence of edges that actually clog and make the filter nonpermeable, which depends on the flow conditions as well as the width of the channels, is, in general, much shorter than the feasible sequences of edges with maximum length.

In this section, we will restrict our attention to a subclass of filters for which we will show how to select the width of the channels so that, as the suspension flows, the feasible sequence of edges that is realized and makes the filter nonpermeable will indeed have maximum length.

\subsection{Subclass of filters. The geometries.}

DEFINITION 7.1. We say that a multigraph $G$ that corresponds to one of our filters has geometry of class $\mathcal{A}$ if there is a nonnegative integer $r$ and a sequence of disjoint percolating paths $P_{0}, P_{1}, \ldots, P_{r}$ in $G$ such that, for each $i, 0 \leq i<r$, the following hold:

1. $P_{i}$ is to the left of $P_{i+1}$.

2. There are positive integers $s_{i}$ such that $P_{i}$ and $P_{i+1}$ are joined by $s_{i}+1$ paths that may have only end points in common; i.e., for each $j, 0 \leq j \leq s_{i}$, there is a path $H_{i, j}$ such that (1) $H_{i, j} \cap P_{i}$ is an end point of $H_{i, j}$, (2) $H_{i, j} \cap P_{i+1}$ is the other end point of $H_{i, j}$, and (3) $H_{i, j_{1}}$ and $H_{i, j_{2}}$ may intersect at their end points only if $j_{1} \neq j_{2}$.

3. $G$ is the union of the percolating paths $P_{i}(0 \leq i \leq r)$ and the "crossing" paths $H_{i, j}\left(0 \leq i<r, 0 \leq j \leq s_{i}\right)$.

For convenience, we assume that the paths $H_{i, j}$ are labeled in such a way that $H_{i, j+1}$ is above $H_{i, j}\left(0 \leq i<r, 0 \leq j<s_{i}\right)$. More precisely, $H_{i, j+1}$ is in the closure of the bounded region whose boundary is included in $\left\{y=y_{t}\right\} \cup P_{i} \cup P_{i+1} \cup H_{i, j}$. Note that $H_{i, 0}$ is included in the bottom boundary and $H_{i, s_{i}}$ is included in the top boundary.

Examples of multigraphs that have the geometry of class $\mathcal{A}$ are shown in Figure 12. The percolating paths $P_{i}$ are in solid lines. The crossing paths $H_{i, j}$ are in dashed lines.

7.2. Subclass of filters. The width of the channels. The physical mechanisms that lead to the clogging of channels may be complex and depend on the particular problem under consideration. Here we will assume the following simple rules. Each channel is either thin or thick. Thick channels never clog, and thin channels eventually clog if there is flow through them. 

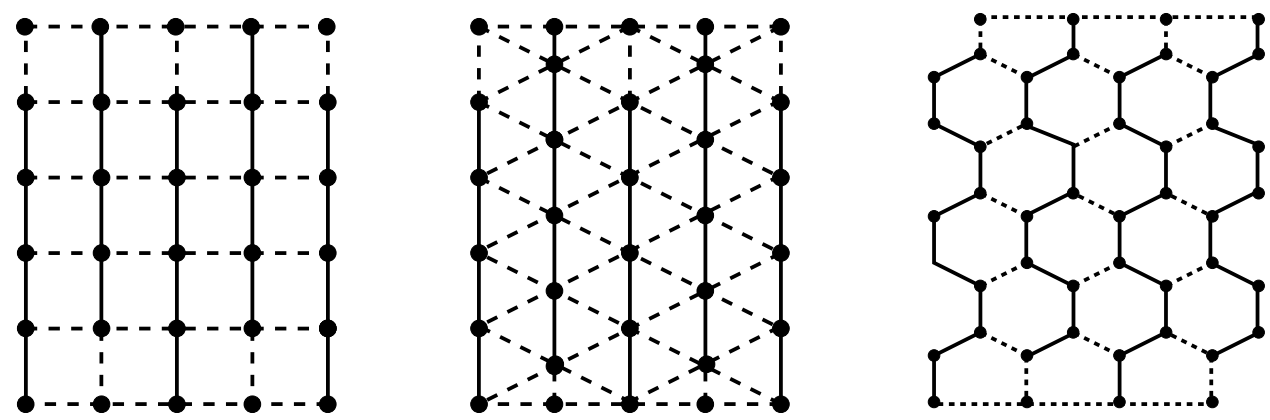

FIG. 13. Multigraphs of Figure 12. The thick edges are in solid lines. Thin edges in dashed lines.

We now select the thin and thick edges. Let $G$ be a multigraph that has geometry of class $\mathcal{A}$. Let $P_{0}, P_{1}, \ldots, P_{r}$ be the percolating paths as in Definition 7.1. Every percolating path is split into subpaths by the crossing paths. For $i$ even, let $\bar{P}_{i}$ be the subpath of $P_{i}$ that contains an exterior node at the top boundary. For $i$ odd, let $\bar{P}_{i}$ be the subpath of $P_{i}$ that contains an exterior node at the bottom boundary. One edge in each of the subpaths $\bar{P}_{i}$ for any $i$ is selected to be thin. One edge in each of the crossing paths $H_{i, j}$ is also selected to be thin. Every other edge is chosen thick. In Figure 13 we show the multigraphs of Figure 12, but now the thick edges are in solid lines and the thin edges in dashed lines.

DEFINITION 7.2. We say that a multigraph $G$ that corresponds to one of our filters is of class $\mathcal{A}$ if $G$ has geometry of class $\mathcal{A}$ and the width of the edges of $G$ are selected as described above.

7.3. The bound realizes for filters of class $\mathcal{A}$. We now show that, for the filters considered in this section, i.e., with multigraph of class $\mathcal{A}$, the bound realizes; i.e., the number of edges that actually clog is equal to our bound, the number of faces of the multigraph of the filter. We show this in two steps. We first show that in each of the paths $\bar{P}_{i}$ the thin edge clogs, and in each of the paths $H_{i, j}$ not included in the top or bottom boundaries, i.e., $H_{i, j}$ for $1 \leq j<s_{i}$, the thin edge also clogs. Then, we show that the number of these paths is equal to the number of faces of the multigraph of the filter.

ObSERVATION 7.1. In each of the paths $\bar{P}_{i}(0 \leq i \leq r)$ the thin edge clogs, and in each of the paths $H_{i, j}$ not included in the top or bottom boundaries $(0 \leq i<r$, $1 \leq j<s_{i}$ ) the thin edge clogs.

Proof. We first note that there is no percolating paths with all thick edges. Thus, the filter eventually ceases to be permeable.

Let $e_{i}$ be the thin edge in $\bar{P}_{i}$. All the other edges in $P_{i}$ are thick, and thus they never clog. As a consequence, while $e_{i}$ is open, the filter is permeable. Thus, $e_{i}$ eventually clogs.

Let $H_{i, j}$ be one of the crossing paths not included in the top or bottom boundaries, i.e., $1 \leq j<s_{i}$. We connect one end point of $H_{i, j}$ to a bottom exterior node and the other to a top exterior node with paths of thick edges as follows. Let $Q$ be the subpath of $P_{i}$ that has an exterior node as an end point, shares the other end point with $H_{i, j}$, and all the edges in $Q$ are thick. Let $R$ be the subpath of $P_{i+1}$ that has an exterior node as an end point, shares the other end point with $H_{i, j}$, and all the edges in $R$ are thick. From the discussion of sections 7.1 and 7.2, it is clear that $Q$ and $R$ are well defined. It is also clear that the union of $Q, R$, and $H_{i, j}$ forms a percolating 


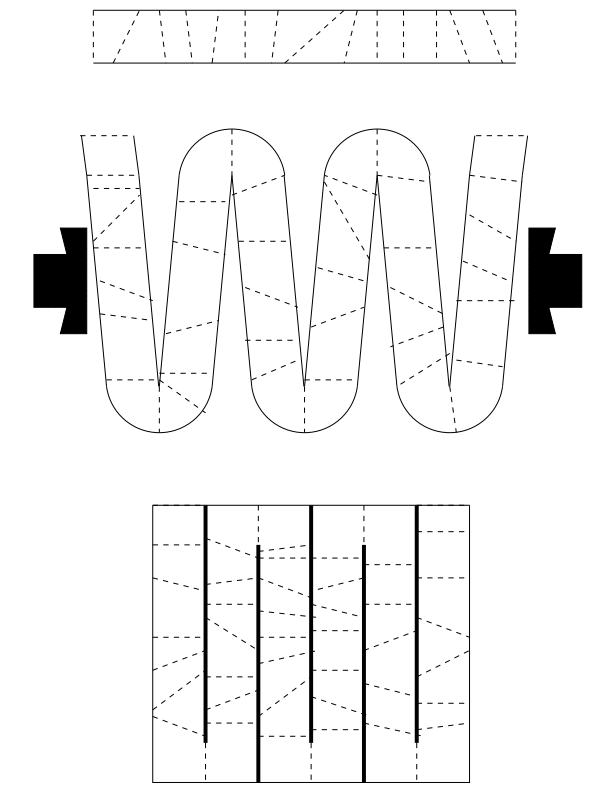

FIG. 14. Building a filter of class $\mathcal{A}$. In the top figure we start with a thin filter with thin channels. In the middle figure we fold the thin filter. The bottom figure shows the resulting filter after folding and compressing. The thick edges are in solid thick lines. The thin edges are in dashed lines.

path whose only thin edge is the one in $H_{i, j}$. Due to the same argument of the last paragraph, this implies that the thin edge in $H_{i, j}$ eventually clogs.

OBSERvation 7.2. The number of the paths $\bar{P}_{i}(0 \leq i \leq r)$ and $H_{i, j}$ not included in the top or bottom boundaries $\left(0 \leq i<r, 1 \leq j<s_{i}\right)$ is equal to $f_{G}$, the number of faces of $G$.

Proof. We first note that the number of the paths $\bar{P}_{i}(0 \leq i \leq r)$ and $H_{i, j}$ not included in the top or bottom boundaries $\left(0 \leq i<r, 1 \leq j<s_{i}\right)$ is equal to $1+r+\sum_{i=0}^{r-1}\left(s_{i}-1\right)=1+\sum_{i=0}^{r-1} s_{i}$. Thus our goal reduces to showing that $f_{G}=1+\sum_{i=0}^{r-1} s_{i}$.

Let $0 \leq i<r$ and $1 \leq j \leq s_{i}$. If we remove from the plane the paths $P_{i}$, $H_{i, j-1}, P_{i+1}$, and $H_{i, j}$, we are left with one bounded and one unbounded connected component. Let $F_{i, j}$ be the bounded component. It is clear that the bounded faces of $G$ are $F_{i, j}$ for $0 \leq i<r$ and $1 \leq j \leq s_{i}$. Therefore, $f_{G}=1+\sum_{i=0}^{r-1} s_{i}$, which completes the proof.

7.4. Building filters of class $\mathcal{A}$ from thin filters. We now discuss a possible means, at least theoretically, to construct filters of class $\mathcal{A}$.

We start with a thin filter with thin channels. By a thin filter we mean that the corresponding graph is the union of disjoint percolating paths connecting the top and bottom boundaries. An example is shown in the top figure of Figure 14.

Next, we fold the thin filter as shown in the middle figure of Figure 14. As we compress the folded filter from the sides, the spaces between folds become the thick channels, and we are left with the new filter shown in the bottom figure of Figure 14. The applied pressure in the folding step should be high, but not so high so the new pore space, the thick channels, are in fact thicker than the channels in the original 
thin filter before folding. The resulting filter is a filter of class $\mathcal{A}$.

Note that the above discussion suggests a way to construct filters of long life even if the original thin filter does not strictly satisfy the condition of having the corresponding graph be the union of disjoint percolating paths.

7.5. Further comments on filters of class $\mathcal{A}$. As channels clog, the permeability of the filter decreases. This is unavoidable. Nevertheless, we expect that this decrease in permeability will be relatively slow (as compared with other filters) for filters of class $\mathcal{A}$, because the suspension can flow with relative ease along the thick channels and, as shown in the proof of Observation 7.1, this family of filters has lots of percolating paths where all but one edge are thick.

\section{REFERENCES}

[1] S. L. Bryant, D. W. Mellor, And C. A. Cade, Physically representative network models of transport in porous media, AIChE J., 39 (1993), pp. 387-396.

[2] V. N. Burganos, C. A. Paraskeva, and A. C. Payatakes, Three-dimensional trajectory analysis and network simulation of deep bed filtration, J. Colloid Interf. Sci., 148 (1992), pp. $167-181$.

[3] H. C. Chan, S. C. Chen, And Y. I. Chang, Simulation: The deposition behavior of Brownian particles in porous media by using the triangular network model, Sep. Purif. Technol., 44 (2005), pp. 103-114.

[4] F. Civan, Reservoir Formation Damage, Gulf Publishing Company, Houston, TX, 2000.

[5] G. Dagan, Flow and Transport in Porous Formations, Springer-Verlag, Berlin, 1989.

[6] S. Datta And S. Redner, Gradient clogging in depth filtration, Phys. Rev. E, 58 (1998), pp. 1203-1206.

[7] R. Diestel, Graph Theory, 3rd ed., Springer, Berlin, 2005.

[8] E. C. Donaldson, B. A. Baker, And H. B. Carrol, Particle transport in sandstones, SPE paper 6905, presented at the 52nd annual fall meeting of the SPE of AIME (Denver, CO), 1977.

[9] F. A. L. Dullien, Porous Media. Fluid Transport and Pore Structure, 2nd ed., Academic Press, New York, 1992.

[10] I. FATT, The network model of porous media-I. Capillary pressure characteristics, Trans. Am. Inst. Min. Engrs., 207 (1956), pp. 144-159.

[11] I. FATT, The network model of porous media-II. Dynamic properties of a single size tube network, Trans. Am. Inst. Min. Engrs., 207 (1956), pp. 160-163.

[12] I. FATT, The network model of porous media-III. Dynamic properties of networks with tube radius distributions, Trans. Am. Inst. Min. Engrs., 207 (1956), pp. 164-181.

[13] G. Grimmett, Percolation, 2nd ed., Springer, Berlin, 1999.

[14] J. H. D. Hampton, S. B. Savage, And R. A. L. Drew, Computer modeling of filter pressing and clogging in a random tube network, Chem. Eng. Sci., 48 (1993), pp. 1601-1611.

[15] J. P. Herzig, D. M. LeClerc, AND P. Le GofF, Flow of suspensions through porous mediaApplication to deep filtration, Ind. Eng. Chem., 62 (1970), pp. 8-35.

[16] A. O. Imdakm and M. Sahimi, Transport of large particles in flow through porous media, Phys. Rev. A, 36 (1987), pp. 5304-5309.

[17] A. O. Imdakm And M. SAHImi, Computer-simulation of particle-transport processes in flow through porous media, Chem. Eng. Sci., 46 (1991), pp. 1977-1993.

[18] J. M. Montgomery, Water Treatment Principles and Design, John Wiley \& Sons, New York, 1985.

[19] K. C. Khilar and H. S. Fogler, Migration of Fines in Porous Media, Kluwer Academic Publishers, Dordrecht, The Netherlands, 1998.

[20] Y. S. Kim AND A. J. Whittle, Filtration in a porous granular medium: 2. Application of bubble model to 1-D column experiments, Transp. Porous Media, 65 (2006), pp. 309-335.

[21] J. Lee And J. Koplik, Network model for deep bed filtration, Phys. Fluids, 13 (2001), pp. 1076-1086.

[22] B. E. Logan, Environmental Transport Processes, John Wiley \& Sons, New York, 1999.

[23] J. D. Logan, Transport Modeling in Hydrogeochemical Systems, Springer-Verlag, New York, 2001.

[24] L. M. McDowell-Boyer, J. R. Hunt, And N. Sitar, Particle transport through porous media, Water Resource Res., 22 (1986), pp. 1901-1921. 
[25] S. D. Rege And H. S. Fogler, A network model for deep bed filtration of solid particles and emulsion drops, AIChE J., 34 (1988), pp. 1761-1772.

[26] J. N. Ryan And M. Elimelech, Colloid mobilization and transport in groundwater, Colloids Surfaces A, 107 (1996), pp. 1-56.

[27] M. Sahimi, Applications of Percolation Theory, Taylor \& Francis, London, 1994.

[28] M. Sahimi, G. R. Gavalas, and T. T. Tsotsis, Statistical and continuum models of fluid-solid reactions in porous media, Chem. Eng. Sci., 45 (1990), pp. 1443-1502.

[29] B. J. Suchomel, B. M. Chen, And M. B. Allen, Network model of flow, transport and biofilm effects in porous media, Transp. Porous Media, 30 (1998), pp. 1-23.

[30] K. E. Thompson And H. S. FogLer, Modeling flow in disordered packed beds from pore-scale fluid mechanics, AIChE J., 43 (1997), pp. 1377-1389.

[31] D. Tiab and E. C. Donaldson, Petrophysics, Gulf Publishing Company, Houston, TX, 1996.

[32] C. Tien and A. Payatakes, Advances in deep bed filtration, AIChE J., 25 (1979), pp. 737-759. 